\title{
Seguimiento de una cohorte de mujeres trabajadoras en cultivos de flores de la Sabana de Bogotá
}

\author{
Marcela E. Varona ${ }^{1}$, Fernando de la Hoz ${ }^{1}$, Germán E. Peña ${ }^{1}$, Omayda Cárdenas ${ }^{1}$
}

\begin{abstract}
Resumen
La presente investigación es un estudio descriptivo sobre las mujeres encontradas pertenecientes a la cohorte de trabajadoras en cultivos de flores de la Sabana de Bogotá, estudiadas por el Grupo de Sanidad del Ambiente del Instituto Nacional de Salud hace 20 años.

Se obtuvo información nueva de las mujeres que se lograron ubicar; se describieron las características generales de dicha población; se determinó la similitud entre la población encontrada y la no encontrada y se estableció la frecuencia y tipo de cáncer desarrollado en las mujeres. Se encontraron $1.218(23,1 \%)$ mujeres del total de mujeres estudiadas en la cohorte inicial, con un promedio de edad de 44,5 años y un tiempo total de trabajo en cultivos de flores de 15 años en promedio, siendo las actividades que ocupan el mayor número de personas, las identificadas como de alta exposición a plaguicidas.
\end{abstract}

Follow-up of a group of women working in floriculture on the Sabana de Bogotá

The present document is a descriptive study of women found working in floriculture on the Sabana de Bogotá (the flat plain surrounding Bogotá), who had been studied by the Instituto Nacional de Salud's Environmental Sanitation group for 20 years.

New information has been obtained about the women, the said population's general characteristics are described, the similarity between the population encountered and not encountered was determined and the frequency and type of cancer which develops in women was established. The study covered 1,218 $(23.1 \%)$ women from the total of women studied in the initial cohort, with an average of 44.5 years of age and an average of 15 years working full-time in flower growing, the activity which occupies the greatest number of people in this area, the women having been identified as being at very high risk of exposure to pesticides.

Significant statistical differences were found in some variables when comparing the population of women encountered with that of the women not encountered. The levels of cancer found in the sample, are much lower than those reported by the Population Cancer Register (Registro Poblacional de Cáncer) in Cali; the proportion of pesticides used in this activity is high and extremely toxic.

${ }^{1}$ Laboratorio de Salud Ambiental, Instituto Nacional de Salud.

Recibido para publicación: febrero 3, 1998 
Desde la aparición del DDT hace más de 40 años, el uso de los compuestos agroquímicos se ha venido incrementando en todo el mundo. Se han utilizado principalmente en la agricultura para mejorar la calidad de los alimentos y en la salud pública para el control de vectores de enfermedades epidémicas como la malaria.

La exposición a los agroquímicos produce en el ser humano algunos efectos sobre la salud que van desde intoxicaciones agudas hasta condiciones crónicas severas.

En nuestro medio ha sido bastante limitada la información sobre la exposición a estos elementos. Contamos, sin embargo, con el estudio realizado hace 20 años por Restrepo et al. (1, 2), quienes determinaron la frecuencia de efectos adversos en la reproducción de mujeres trabajadoras en cultivos de flores de la Sabana de Bogotá. Esta cohorte de mujeres representa la única pobiación conocida del país con la que se pudieran estudiar los efectos crónicos de la exposición ocupacional a plaguicidas. Sin embargo, ubicar nuevamente a esta cohorte de mujeres representa un trabajo difícil ya que después de 20 años, las mujeres han podido cambiar de trabajo, de localidad o pudieron haber fallecido; además, la información que se pueda obtener de estas mujeres en gran parte dependerá de su recordatorio.

Con este proyecto de investigación se pretende obtener información nueva de las mujeres del estudio de Restrepo et al., describiendo las características y evaluando la representatividad de las mujeres que se logren captar.

\section{Marco téorico}

El uso de los plaguicidas sigue siendo en la actualidad el método más utilizado para mejorar el rendimiento de la producción agrícola. Estos compuestos, después de cumplir su objetivo primario, se dispersan en el medio ambiente biodegradándose o bioacumulándose de acuerdo con sus características fisicoquímicas (3).

La exposición a los agroquímicos genera una gran variedad de efectos tanto agudos como crónicos, entre los que se incluyen: neurotoxicidad (insecticidas, fungicidas); daño pulmonar (paraquat); lesiones hepáticas y renales (sales de hierro y cobre, mercurio inorgánico); lesiones irritativas o cáusticas locales (arsenicales); anormalidades inmunológicas y alteraciones reproductivas (organoclorados) (4-6). Numerosos estudios han informado un elevado riesgo de desarrollo de cáncer en la población que labora directamente con plaguicidas en agricultura y en cultivo de flores (7-14). Entre éstos, se encuentran principaimente leucemias, linfomas no Hodgkin, mieloma múltiple, sarcomas de tejidos biandos, cáncer de seno, ovario, pulmón, hígado y cerebro. No se conocen con certeza los mecanismos por los cuales los plaguicidas pueden desarrollar cáncer.

En Colombia, el consumo de plaguicidas para uso agrícola en los últimos cinco años, ha variado entre 20.000 y 25.000 toneladas de ingredientes activos, cifra que aumenta de manera importante al sumar los de uso doméstico y pecuario. Dentro de los países de Latinoamérica, Colombia es el tercer país consumidor de plaguicidas, después de Brasil y México.

En la Sabana de Bogotá, se han estado utilizando más de cien productos comerciales de plaguicidas, que contienen cerca de cincuenta ingredientes activos diferentes entre los que se destacan los organoclorados, los organofosforados y los carbamatos (15).

Todo lo anterior resalta la importancia de realizar estudios epidemiológicos sobre la exposición a plaguicidas y los efectos que causan sobre la salud. La cohorte de mujeres que fue localizada hace 20 años reune las características para ser estudiada nuevamente, ya que una parte ha estado expuesta por largo tiempo a los plaguicidas.

Hace 20 años, Restrepo et al $(1,2)$ realizaron un estudio en más de 5.000 mujeres que trabajaban en los cultivos de flores de la Sabana de Bogotá. En este estudio se determinó la prevalencia de efectos adversos sobre la reproducción en mujeres trabajadoras en los cultivos de flores. Un moderado incremento en la prevalencia de abortos, niños prematuros y malformaciones congénitas fue detectado en los embarazos ocurridos después de la exposición en los cultivos de flores. 
El segundo estudio, de casos y controles, basado en el anterior, evaluó los efectos de nacimiento de los hijos de las madres expuestas a plaguicidas. El análisis de los casos y controles incluyó 222 niños con defectos de nacimiento y 443 normales; se encontró un incremento de hemangiomas en niños cuyos parientes estaban expuestos a plaguicidas en la industria de flores.

\section{Objetivos}

El objetivo general de este estudio fue describir las características generales de las mujeres pertenecientes a la cohorte de mujeres estudiadas hace 20 años por Restrepo et al. y que se logren ubicar en la actualidad.

Determinar la similitud entre la población encontrada y la no encontrada.

Establecer la frecuencia y tipo de cánceres desarrollados en la población encontrada.

\section{Metodología}

Esta investigación es un estudio descriptivo sobre las mujeres encontradas, pertenecientes a la cohorte de trabajadoras en cultivos de flores estudiadas por el Grupo de Sanidad del Ambiente del Instituto Nacional de Salud hace 20 años (1, 2). Dicho grupo cuenta con las bases de datos que incluyen las historias clínicas y los datos de identificación de las mujeres que participaron en el estudio, usadas para su ubicación posterior. Con esta información, se realizó una búsqueda activa de cada una de las 5.274 mujeres, ya fuera en las empresas de cultivos de flores o en sus viviendas $\mathrm{y}$, en algunos casos, cuando la mujer hubiera fallecido, se ubicaron los familiares cercanos.

Para localizar a las mujeres, se zonificaron 30 localidades en la Sabana de Bogotá y se empleó ayuda de las alcaldías, las oficinas de planeación y los censos de población nacional; también fue necesario recurrir a registros hospitalarios y de centros de salud con la ayuda de los promotores de salud locales. Se contactaron algunas de las empresas que participaron en el estudio inicial, hace 20 años, a las que se les solicitó un listado de las mujeres mayores de 30 años. Parte de las empresas que suministraron esta información contaban con mujeres que laboraban aún en éstas. Se estableció comunicación con el médico de salud ocupacional de cada empresa contactada, con el fin de dar a conocer los objetivos del estudio y así proceder a realizar la recolección de la información. De la misma manera, antes de aplicar la encuesta a las mujeres o a sus familiares se les explicó claramente en que consistía el estudio y una vez ellas accedían participar voluntariamente firmaban un consentimiento escrito.

Un grupo de encuestadores se trasladó a cada una de las localidades aplicando un cuestionario que fue diseñado para recoger la información que incluyó las siguientes variables: nombre, edad, lugar de residencia, tiempo y tipo de trabajo, empresas de flores para las que trabaja o trabajó, otros oficios o trabajos con sustancias tóxicas como solventes orgánicos o disolventes, cadmio, plomo y cromo, historia reproductiva, antecedentes de tabaquismo y en los casos de cáncer, fecha y organismo donde se realizó el diagnóstico. Un profesional revisó aleatoriamente el $10 \%$ de la captura de los datos cada semana.

Los encuestadores fueron previamente entrenados y con el objeto de hacer los ajustes necesarios tanto de instrumentos como de tiempos y movimientos, se llevó a cabo un pilotaje de esta encuesta aplicándose a una selección de trabajadores de planta del Instituto Nacional de Salud.

También se diseñó y aplicó una encuesta dirigida a las empresas de floriculturas donde se encontraron mujeres que aún están laborando, con la que se obtuvieron datos sobre el tamaño de la empresa (pequeña: menos de 100 trabajadores, mediana: entre 100 y 400 trabajadores, grande: mayor de 400 trabajadores), el tipo y la cantidad de plaguicidas utilizados y la totalidad de área en hectáreas cultivadas.

La exposición a plaguicidas se dividió en categorías de acuerdo con el tipo de trabajo en los cultivos de flores, así: alta (las que trabajaron en cultivo y corte/clasificación y empaque) y baja exposición (administración, construcción y mantenimiento, preparación de terreno y banco de plantas madre).

Los diagnósticos de cáncer y las causas de defunción en las mujeres fallecidas fueron 
corroborados con la historia clínica o con el certificado de defunción correspondiente, cuando se pudo acceder a esta información.

El procesamiento de toda la información se realizó con el paquete estadístico Epi-Info 6.03.

Se contó además, con la base de datos de Restrepo et al., con información de las mujeres encuestadas hace 20 años.

Para la caracterización de la población, se obtuvieron frecuencias simples de las variables. A las variables continuas se les estimó la media y la desviación estándar. Al resto de variables se les calcularon sus proporciones.

Utilizando la base de datos de Restrepo et al., se compararon las medias de algunas variables de la muestra de mujeres encontradas con las mujeres no encontradas, utilizando el estadístico $F$ y estimando el valor de $p$, definiendo una significancia estadística con un valor de $p<0,05$.

Finalmente, se compararon las tasas de incidencia encontradas en la población estudiada (1.218 mujeres) con las registradas por el Registro Poblacional de Cáncer de Cali (16). Para determinar las tasas de incidencia de la población estudiada, se utilizó el indicador años personas,

Cuadro 1. Características de las 1218 mujeres encontradas pertenecientes a una cohorte de mujeres trabajadoras en las floriculturas de la Sabana de Bogotá, 1996

\begin{tabular}{lcc}
\hline Variables & Media & DE \\
\hline Edad en años & 44,5 & $\pm 8,5$ \\
Años en floricultura & 15,0 & $\pm 6,1$ \\
Edad de menarquia & 13,7 & $\pm 1,6$ \\
Edad de primera gestación & 21,3 & $\pm 4,7$ \\
Número de gestaciones & 3,9 & $\pm 2,3$
\end{tabular}

donde cada trabajador aportó una determinada cantidad de años según su trabajo en cultivos de flores.

\section{Resultados}

Se encontraron y entrevistaron un total de 1.218 $(23,1 \%)$ mujeres, siendo la mayoría proveniente de Bogotá (20\%), Facatativá (14\%), Funza (14\%) y Madrid (13\%). Se encontró en las mujeres un promedio de edad de 44,5 años. El tiempo total de trabajo en cultivos de flores fue de 15 años en promedio con variaciones entre 1 y 41 años. Las actividades que ocupan mayor número de personas durante más tiempo dentro de los cultivos son las identificadas como de alta exposición a plaguicidas (cultivo y corte, clasificación y empaque). Hoy en día, 576 mujeres $(47,3 \%)$ continúan trabajando en floriculturas. Las características generales de las mujeres entrevistadas durante este estudio se presentan en la cuadro 1.

Entre otros antecedentes, se encontró una edad media de la menarquia de 13,8 años, un promedio de 3,9 gestaciones por mujer y una edad de 21,3 años al momento del primer embarazo. El 26,6\% afirmaron haber fumado alguna vez en su vida.

Para comparar la población de mujeres encontradas con las no encontradas, se utilizó la base de datos original de Restrepo et al. (cuadro 2). Se hallaron diferencias estadísticamente significativas en algunas variables de importancia como el tiempo de estar trabajado en floricultura, el tiempo de laborar en la empresa actual, la edad y los años de escolaridad.

Cuadro 2. Comparación de algunas variables entre las 1.218 mujeres encontradas y las no encontradas de la cohorte de mujeres trabajoras en los cultivos de flores de la Sabana de Bogotá, estudiada hace 20 años.

\begin{tabular}{|c|c|c|c|c|c|c|c|}
\hline \multirow[b]{2}{*}{ Variable } & \multicolumn{2}{|c|}{ Encontradas } & \multicolumn{5}{|c|}{ No encontradas } \\
\hline & $\mathrm{n}$ & Media & DE & $n^{*}$ & Media & $\mathrm{DE}$ & p \\
\hline \multicolumn{8}{|l|}{ Tiempo de trabajo (años) } \\
\hline & $<0,001$ \\
\hline trabajo en floricultura & 1.218 & 3,65 & 2,7 & 4.077 & 3,05 & 3,1 & $<0,001$ \\
\hline Edad (años) & 1.218 & 29,07 & 8,5 & 4.077 & 27,1 & 7,8 & $<0,001$ \\
\hline Años de escolaridad & 1.218 & 2,21 & 0,6 & 4.077 & 2,26 & 0,6 & $<0,05$ \\
\hline
\end{tabular}

*Nota: en 21 casos no se logró establecer la identificación de la mujer mediante esta comparación. 
Como se muestra en el cuadro 3 , se encontraron 31 casos de cáncer: 15 en mujeres vivas y 16 entre las fallecidas. Otras 13 mujeres murieron por causas diferentes al cáncer (4 por evento cerebrovascular, 3 por infarto agudo del miocardio, 2 por trauma, 2 por enfermedades del colágeno y 2 sin información sobre la causa de muerte), para un total de 29 mujeres fallecidas. Sólo se pudo confirmar el diagnóstico de cáncer en $65 \%$ de las mujeres.

Cuadro 3. Tipos de cáncer en las 1.218 mujeres encontradas, pertenecientes a una cohorte de mujeres trabajadoras en la Sabana de Bogotá estudiada hace 20 años.

\begin{tabular}{lc}
\hline \multicolumn{1}{c}{ Localizaciones } & Número \\
\hline Uterino & 14 \\
Gástrico & 6 \\
Sin información & 3 \\
Sangre/hueso & 2 \\
Pulmón & 2 \\
Otros (ovario, cuello) & 2 \\
Sistema nervioso central & 1 \\
Seno & 1 \\
\hline Total & $\mathbf{3 1}$ \\
\hline
\end{tabular}

En el cuadro 4 se comparan las tasas de cáncer encontradas en la muestra y las registradas por el Registro Poblacional de Cáncer de Cali para mujeres entre 35 y 69 años de edad, y se encontró que las mujeres pertenecientes a la muestra del estudio presentan una tasa de incidencia de cáncer más bajo.

Cuadro 4. Comparación de las tasas de incidencia de las 1.218 mujeres encontradas con las de Santiago de Cali, Valle.

\begin{tabular}{lc}
\hline Población & Tasa de incidencia de cáncer \\
\hline $\begin{array}{l}\text { Cali } \\
\text { Mujeres encontradas (1.218) }\end{array}$ & $169 \times 100.000$ años/persona \\
\hline
\end{tabular}

Nota: Las de Santiago de Cali se estimaron a partir de los datos registrados por el Registro Poblacional de Cáncer de Cali para mujeres entre 30 y más años durante el quinquenio 1986-1991, para todos los cánceres (excepto el cáncer de piel); se presenta la tasa anual promedio.

Sólo se estableció el tipo de plaguicida usado en la empresa donde laboraba la mujer en 120 trabajadoras (10\%). De éstas, $43 \%$ trabajó en cultivos de flores donde se usaban plaguicidas catalogados como alta o extremadamente tóxicos (ver anexo); se identificó el uso de 44 plaguicidas diferentes. El porcentaje de mujeres expuestas a otros productos como solventes orgánicos y metales pesados fue mínimo $(2,8 \%)$.
De las 62 empresas de flores que participaron en el estudio inicial hace 20 años, se logró ubicar 32 empresas de las cuales se visitaron 7 donde aún se encuentran laborando mujeres. De la encuesta a estas empresas, se encontró que $85,7 \%$ correspondía a una empresa de tamaño mediano y $14,3 \%$ a empresas grandes. La cantidad de hectáreas cultivadas en promedio en las empresas de tamaño mediano es de 12,4 y en la empresa grande el total de hectáreas cultivadas es de 70 .

\section{Discusión}

La evidencia epidemiológica que existe para determinar alguna asociación entre la exposición a plaguicidas y cáncer es bastante limitada. Existen pocos estudios analíticos (casos y controles y cohortes) que prueben tal asociación. Entre éstos se incluye algunos que, con alguna fortaleza metodológica, asocian la exposición a plaguicidas con cáncer del sistema nervioso central $(3,8)$.

En la población de mujeres encontradas, se hallaron diferencias estadísticamente significativas al compararla con algunas características de la cohorte estudiada hace 20 años. Esto significa que los resultados de la incidencia de cáncer encontrados no sean generalizables al resto de la cohorte y podrían existir algunos sesgos de selección en la población de estudio que afectarían el análisis para buscar posteriormente una asociación entre uso de plaguicidas y cáncer. El sesgo de selección en nuestra población debería operar sobrestimando una asociación que fuera más débil en la cohorte de donde esta población procede. Al ser este un estudio descriptivo, no se pretende buscar dicha asociación.

Se encontró que la mitad de las mujeres de las 1.218 encontradas continuaban trabajando en cultivos de flores lo cual puede introducir el sesgo del trabajador sano, es decir, que las personas que continúan trabajando en cultivos de flores podrían haber tenido un mejor estado de salud que quienes se retiraron de la actividad.

Entre los principales inconvenientes del estudio está la carencia de buenas herramientas para medir los eventos, lo que puede haber introducido 
sesgos de clasificación. La forma de recolección de los datos se basa en la información suministrada por las trabajadoras sobre sus labores desde hace 20 años. Esto implica que no sea posible establecer con validez la exposición a los plaguicidas y la presencia o ausencia de algún tipo de cáncer. Los cambios en la actividad, los plaguicidas usados en las empresas, el lugar de trabajo y las diferencias en las medidas de higiene y seguridad industrial hacen difícil establecer el tipo (alta y baja exposición) y el tiempo de exposición a un plaguicida específico. A pesar que los diagnósticos de cáncer referidos en las encuestas fueron corroborados con historias clínicas y registros de defunciones, se deben tener en cuenta las deficiencias que estos últimos presentan para establecer un diagnóstico certero.

Existen metodologías que permiten medir con validez la exposición a los plaguicidas pero son costosas y técnicamente complejas.

Un factor que no fue tomado en consideración en el estudio fue el uso de elementos de protección y las medidas de higiene y seguridad industrial, que podrían tener un efecto protector al determinar los daños que sobre la salud producen los plaguicidas.

El Registro Poblacional de Cáncer de Cali actualmente se puede considerar como la fuente de información de incidencia de cáncer más confiable de Colombia. Por esta razón, se compararon las tasas de incidencia de cáncer de la población del estudio con la de este registro, conociendo de antemano que las de este último no pueden ser generalizables a otras poblaciones que tengan características diferentes a las de la población caleña. Se halló, sin embargo, que la tasa de incidencia de cáncer de las mujeres trabajadoras en cultivos de flores no fue superior a la informada por el registro de Cali.

\section{Conclusiones}

El principal hallazgo del estudio consistió en determinar que las características generales de la población de mujeres encontradas difieren con significancia estadística de la cohorte estudiada por Restrepo et al. por lo que no es posible inferir nuestros resultados sobre esta última.
Los instrumentos usados para medir retrospectivamente la exposición a plaguicidas y los eventos de la salud en las mujeres estudiadas tienen algunas limitaciones. Los estudios en el futuro deben considerar aspectos técnicos y económicos que permitan establecer estos parámetros con mayor precisión y validez.

\section{Recomendaciones}

Es bien conocida la asociación estadística existente entre la exposición a plaguicidas y el desarrollo de diferentes tipos de cáncer, además de otros problemas sobre la salud. Aunque el recordatorio de las mujeres encuestadas no es confiable, se determinó que una alta proporción de los plaguicidas usados en esta actividad son alta y extremadamente tóxicos a pesar que se han desarrollado plaguicidas de ligera toxicidad. Por tanto, es de suma importancia que las empresas de cultivos de flores establezcan programas de salud ocupacional que orienten a los trabajadores sobre las medidas de higiene y seguridad industrial que deben seguir. Sería de gran interés estudiar el uso actual de los plaguicidas en los cultivos de flores y las medidas de higiene y seguridad industrial que se siguen al respecto.

Dado el riesgo elevado para desarrollar cáncer que presentan los trabajadores que manejan plaguicidas, las ARP deben incluir programas de tamizaje y diagnóstico temprano del cáncer, especialmente para los cánceres de cérvix y seno; deben establecer un sistema de vigilancia epidemiológica de la ocurrencia de estos eventos y más estudios sobre la clasificación de la exposición.

Un método alternativo para evaluar el efecto de la exposición a plaguicidas sobre la salud es mediante buenos sistemas de vigilancia epidemiológica de abortos espontáneos y malformaciones congénitas, así como la ocurrencia de cáncer a través de métodos de tamizaje y detección temprana, análisis anual de las incapacidades en estas empresas y notificación obligatoria de los pacientes con tumores.

\section{Agradecimientos}

Queremos expresar nuestros más sinceros agradecimientos a todo el equipo que participó 
en el trabajo de campo necesario para el desarrollo de la presente investigación y a todo el personal administrativo que labora en el Laboratorio de Salud Ambiental.

Igualmente al doctor Gabriel Carrasquilla, integrante del Comité Técnico Científico del Instituto Nacional de Salud por sus valiosos aportes en la fase final de la investigación; al Instituto de Seguros Sociales, Administradora de Riesgos Profesionales, por el apoyo financiero dado para la realización del estudio; a las empresas de flores que participaron y a la Asociación Colombiana de Exportadores de Flores (ASOCOLFLORES), por su cooperación.

\section{Referencias}

1. Restrepo M, et al. Birth defects among children born to a population ocupationally exposed to pesticides in Colombia. Scand J Work Environ Health 1990;16:239-46.

2. Restrepo M, et al. Prevalence of adverse reproductive outcomes in a population ocupationally exposed to pesticides in Colombia. Scand J Work Environ Health 1990;16:232-8.

3. Burger M, et al. Plaguicidas en el medio ambiente. Montevideo: Universidad de la República, 1991.

4. Fernández G. Intoxicación por plaguicidas. Montevideo: Editorial Monteverde, 1970.

5. Figa-Talamanca I, Mearelli I, Valente P. Mortality in a cohort of pesticide applicators in an urban setting. Int $\mathrm{J}$ Epidemiology 1993;22:674-6.

6. Weisenburger $D$. Human health effects of agro-chemical use. Human Pathol 1993;24:571-6.

7. Faustini A, et al. Cohort study of mortality among farmers and agricultural workers. Med Lav 1993;84: 31-41.

8. Figa-Talamanca I, et al. Cancer mortality in a cohort of rural licensed pesticide users in the province of Rome. Int J Epidemiology 1993;22:579-83.

9. Heineman EF, Gao YT, Dosemeci M, McLaughlin JK. Occupational risk factors for brain tumors among women in Shanghai, China. JOEM 1995;37:288-93.

10. Littorin M, Attewell $\mathbf{R}$, Skerfving $\mathbf{S}$, et al. Mortality and tumour morbidity among Swedish market gardeners and orchardists. Int Arch Occup Environ Health 1993;65:163-69.

11. Savitz DA, Andrews KW, Brinton LA. Occupation and cervical cancer. JOEM 1995;37(3):357-61.

12. Ciccone G, Mirabelli D, Levis A, et al. Myeloid leukemias and myelodysplastic syndromes: chemical exposure, histologic subtype and cytogenetics in a casecontrol study. Cancer Genet 1993;68:135-9.

13. Hoar S, et al. The role of agricultural pesticide use in the development of non-Hodgking's lymphoma in women. Arch Environ Health 1993;48:353-8.

14. Hayes W. Pesticides studied in man. Baltimore, London: Williams \& Wilkins, 1982.

15. Morales C. Los plaguicidas agrícolas en Colombia. Bogotá: Ministerio de Agricultura, Instituto Colombiano Agropecuario; 1992.

16. Registro Poblacional de Cáncer de Cali, Departamento de Patología, Universidad del Valle. Documento preparado por Edwin Carrascal, Director.

Anexo 1. Distribución del uso de los plaguicidas según grupo y toxicidad.

Distribución de los plaguicidas según grupo

\begin{tabular}{lcc}
\hline Grupo & $\mathbf{n}$ & $\%$ \\
\hline Insecticida simple & 110 & 52,4 \\
Fungicida simple & 53 & 25,2 \\
Acaricida & 24 & 11,4 \\
Herbicida simple & 12 & 5,7 \\
Molusquicida & 4 & 1,9 \\
Nematicida & 3 & 1,4 \\
Desinfectante del suelo & 2 & 1,0 \\
Regulador fisiológico & 1 & 0,5 \\
Coadyuvante & 1 & 0,5 \\
\hline Total & $\mathbf{2 1 0}$ & $\mathbf{1 0 0}$
\end{tabular}

Según clasificación por grupos de los plaguicidas, Ministerio de Salud, 1995.

Distribución de los plaguicidas según su toxicidad

\begin{tabular}{lcc}
\hline Grupo & $\mathbf{n}$ & $\%$ \\
\hline Extremadamente tóxico & 64 & 30,4 \\
Altamente tóxico & 31 & 14,8 \\
Medianamente tóxico & 86 & 41,0 \\
Ligeramente tóxico & 29 & 13,8 \\
\hline Total & $\mathbf{2 1 0}$ & $\mathbf{1 0 0}$
\end{tabular}

Según clasificación toxicológica de los plaguicidas, Ministerio de Salud, 1995. 